\title{
EDUCATIONAND TRAINING Patient and public understanding of the concept of 'personalised medicine' in relation to cancer treatment: a systematic review
}

\author{
Authors: Jed Botham, ${ }^{A}$ Valerie Shilling ${ }^{B}$ and John Jones ${ }^{C}$
}

\section{Personalised medicine (PM) is becoming increasingly integrated into standard clinical practice for treating numerous diseases, including cancer. Implementing PM into healthcare systems will only be successful with the acceptance and input of both patients' and public opinion. This review, therefore, aimed to identify both patients' and public understanding, and perceived benefits and concerns of PM in cancer treatment. A literature search was conducted using MEDLINE, EMBASE, PsycINFO and CINAHL databases. The eligibility criteria specified that papers must explore the public or patients' understanding of PM or pharmacogenomic (PGx) testing in relation to cancer treatment. Patients have a greater understanding of, and trust in, PM compared with members of the public, but often misunderstand how genomic testing in PM works. Key areas that can be targeted to inform future health literacy interventions include genetic literacy for the public and understanding of how PM testing and treatment works for patients.}

KEYWORDS: personalised medicine, patients' understanding, public understanding, cancer, genomics

DOI: 10.7861/fhj.2021-0063

\section{Introduction}

Personalised medicine (PM) represents an extension of traditional approaches to healthcare, incorporating an individual's genetics and genomics in the prevention, diagnosis, stratification and treatment of disease. ${ }^{1}$ Genetics is the study of individual genes and how they can be inherited. Genomics refers to the study of all the genes a person has (their genome) and how they interact with the environment to influence the

Authors: ${ }^{\mathrm{A}} 4$ th year medical student, Brighton and Sussex Medical School, Brighton, UK; ${ }^{B}$ research fellow, Sussex Health Outcomes Research and Education in Cancer (SHORE-C), Brighton, UK; ' ${ }^{\text {senior }}$ lecturer in haemato-oncology and consultant haematologist, Brighton and Sussex Medical School, Brighton, UK development of diseases (such as cancer). The prevention and treatment of cancer is one of the most important areas of PM, considering that half of all people in the UK will be diagnosed with cancer in their lifetime. ${ }^{2}$

According to the Genome UK strategy launched by the UK government in 2020, implementing PM involves 'engagement and dialogue with the public, patients and our healthcare workforce, placing the patient and the diverse UK population at the heart of this journey. ${ }^{11}$ For patients and the public to make informed decisions regarding their care, they need an understanding of PM, particularly what genomic tests look for and the implications test results may have.

The term PM, and what it involves, is still largely unfamiliar to the public. ${ }^{3}$ For patients, PM is complicated by confusing language, including the synonymous use of precision, personalised, stratified and targeted medicine. ${ }^{4}$ These terms are also used in varied healthcare scenarios, from genetic risk screening to genomic testing and treatment. Patient knowledge of PM influences their decision making in relation to treatment and, so, providing adequate education and support is vitally important. $^{5}$

It has been 2 years since a review of public opinion was conducted and there has not been a published systematic review including patient understanding of PM. ${ }^{3}$ This review, therefore, aims to explore both patients' and the public's understanding of PM in relation to cancer treatment, as genomic testing and medicine is set to revolutionise healthcare. With the treatment of cancer at the forefront of adoption and implementation of PM, findings will be able to be shared across specialties. This will help to inform future public engagements and improve the quality of dialogue between patients, their families and healthcare professionals. Without this, the public and patients will be less well-informed in decision making regarding their care and may be wary of the wider implications that genomic medicine may confer.

\section{Aims and objectives}

We aimed to identify what patients and the public understand regarding the use of PM in cancer treatment; learn what patients and the public perceive as the benefits of PM use in cancer treatment; and learn what concerns patients and the public have with the use of PM in cancer treatment. 


\begin{tabular}{|c|c|}
\hline Inclusion & Exclusion \\
\hline $\begin{array}{l}\text { Papers exploring patients' } \\
\text { and public understanding } \\
\text { of PM in relation to } \\
\text { cancer treatment. } \\
\text { Papers that look at } \\
\text { patient or public } \\
\text { understanding of PGx } \\
\text { testing to inform cancer } \\
\text { treatment. } \\
\text { At least one group of } \\
\text { participants are members } \\
\text { of the public or patients } \\
\text { and their results are } \\
\text { disaggregated. }\end{array}$ & $\begin{array}{l}\text { Papers only looking at patients' or } \\
\text { public understanding of predictive } \\
\text { genetic tests for cancer risk genes. } \\
\text { Papers reporting solely on clinical } \\
\text { outcomes of personalised cancer } \\
\text { treatments. } \\
\text { Papers that focus on the } \\
\text { understanding of personalised } \\
\text { treatments used in paediatric } \\
\text { oncology. } \\
\text { Studies that are reviews, conference } \\
\text { abstracts, letters or editorials. } \\
\text { Studies not reported in English. }\end{array}$ \\
\hline
\end{tabular}

\section{Methods}

\section{Search strategy}

The protocol for this review was prospectively registered with PROSPERO (reference CRD42021221971). A literature search was conducted on 13 February 2021 in MEDLINE, EMBASE, PsycINFO and CINAHL. The full search strategy is detailed in supplementary material S1. Eligible studies must have aimed to explore the public or patients' understanding of personalised medicine in relation to cancer treatment or genomic testing to inform cancer treatment. The full list of inclusion and exclusion criteria are shown in Table 1.

\section{Study selection}

A PRISMA flowchart has been produced detailing the study selection process and the reasons for exclusion of each full-text paper (Fig 1). The abstracts and titles of the references retrieved by the electronic searches were screened for relevance by two researchers and duplicates were removed. Full paper copies of potentially relevant studies were then obtained and screened again using the eligibility criteria. The reference lists of all included studies were also screened for potentially relevant articles that met the eligibility criteria. Any discrepancies in selection of articles were resolved by a third researcher.

\section{Data extraction and synthesis}

Data from each selected study was extracted into a standardised data extraction table, including author, year, location, study design, aspect of PM and sample characteristics of the included population. Quality appraisal was conducted using the Mixed Methods Appraisal Tool (MMAT), as described by Hong et al. ${ }^{6}$

The MMAT is a critical appraisal tool, designed for systematic reviews comprising qualitative, quantitative and mixed methods studies. Appraisal involves two screening questions and five methodological quality criteria, which differ depending on study design. These five methodological quality criteria are scored out of one and used to formulate a total score out of five for each study.

This was undertaken by one researcher and independently reviewed

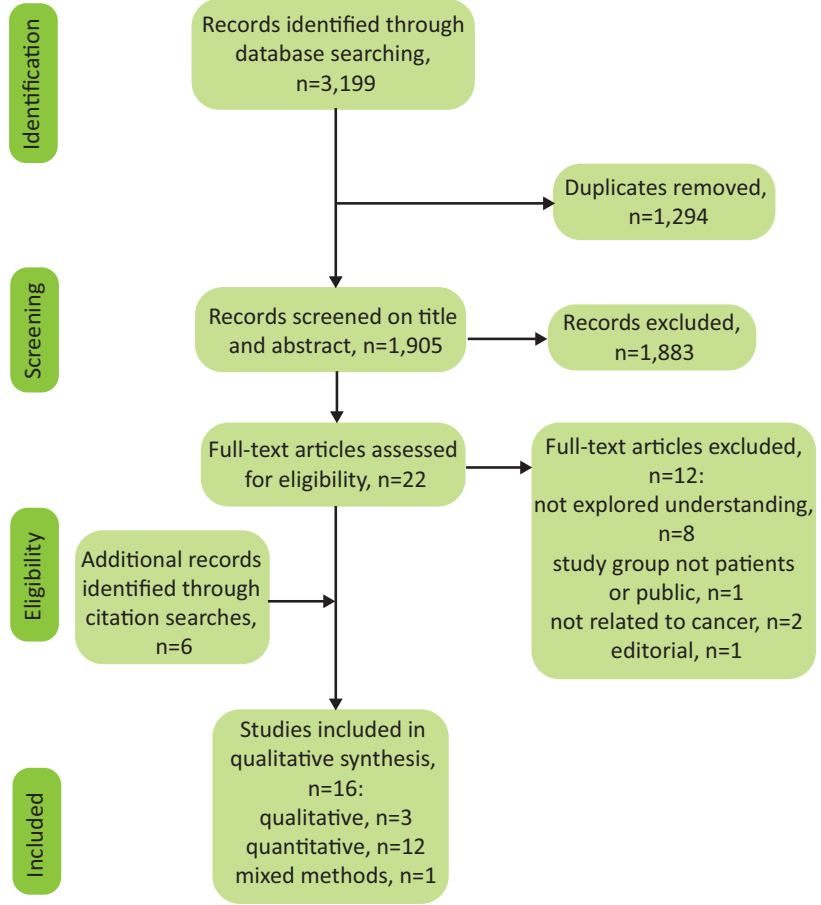

Fig 1. Identification and selection of eligible studies.

by a second. Studies were not excluded based on their MMAT score, instead, the score was used to give an overall impression of the quality of studies identified in the literature search.

\section{Results}

\section{Study characteristics and quality}

A total of 16 papers were included in the review and are summarised in the supplementary material S2. The 16 articles identified cover three main aspects of PM. Five articles focus on gene expression profiling (GEP) in breast cancer, which analyses the activity of a number of genes at once and can be used to inform treatment. Eight papers report on analysis of somatic DNA mutations, which are acquired mutations that can accumulate and lead to the development of cancer. Unlike germline mutations, which can be inherited, somatic mutations are not passed on. Three papers focus on PM in clinical trials that include a number of testing modalities. Only four studies scored highly (5/5) on the MMAT criteria, all of which had clear methodologies and explanation of participant sampling methods. Lower quality studies used convenience sampling of patients attending a single cancer clinic that impacts on the strength of the evidence.

\section{Data synthesis}

Results were synthesised in a descriptive manner in order to provide an overall impression of the findings. This covers key themes of patients' and public understanding, benefits and concerns of PM. Problems with understanding PM related to PM terminology, how PM testing works and the sources of information patients use. No data were available to identify the main sources 
of information the public use to research PM. The main benefits of PM were found to be feeling informed about treatment, the accuracy of tests and a feeling of benefiting others (altruism). Concerns identified include the psychological impact of test results, increased health insurance costs and the number of biopsies needed for testing.

\section{Understanding of terminology}

Five papers reported on patient and public understanding of PM terminology. ${ }^{7-11}$ The concept of PM was understood by $89 \%$ of patients enrolled in clinical trials, $48 \%$ of patients undergoing somatic testing and $27 \%$ of the public. Of patients undergoing somatic testing, $19 \%$ did not include genomic medicine in their definition of PM. ${ }^{8,9}$ The term 'somatic mutation' was correctly defined by $0.7 \%-12 \%$ of patients. 8,10 Only $13 \%$ of patients knew the difference between somatic and germline testing. ${ }^{10,11}$ The concept of 'targeted therapy' was understood by $59 \%-85 \%$ of patients. ${ }^{7,10,11}$

\section{Understanding how PM test works}

Seven papers explored patients' understanding of how PM tests work. ${ }^{7,8,12-16}$ Between $91 \%$ and $92 \%$ of patients were aware that GEP test results can be used to inform chemotherapy decisions. ${ }^{15,16}$ Knowledge that GEP predictions can only be made when the patient is receiving hormone therapy, was reported to be $21 \%-25 \%{ }^{15,16}$ Hormone therapy involves blocking the production of hormones that a person produces, or interfering with the way the hormone interacts with the cancer, in order to inhibit the growth of hormone-sensitive tumours. Between $50 \%-84 \%$ of patients understand that somatic testing can be used to inform treatment. ${ }^{7,8,12,14}$ Less than half of patients $(48 \%)$ reported having sufficient knowledge of how PM works to make an informed decision regarding genomic testing in cancer. ${ }^{12}$

\section{Information seeking}

Six papers identified where patients most commonly seek information regarding PM. ${ }^{11,13-15,17,18}$ Between $27 \%$ and $62 \%$ of patients had sought information regarding PM. ${ }^{11,14}$ The most common sources were the internet and doctors. ${ }^{11,13,14,17,18}$ Ninetyone per cent of patients report their cancer specialist as being the most helpful source of information and $24 \%$ indicated that internet sources were most useful. ${ }^{14}$ Those who sought information performed significantly better on the genetic knowledge test. ${ }^{15}$

\section{Feeling informed about treatment}

Eight papers discussed the ability of PM testing to inform treatment. $9,12,14,15,17,19-21$ Between $57 \%$ and $92 \%$ of patients believed that PM testing aided in decision making regarding treatment. ${ }^{9,12,15,20,21}$ Of patients with breast cancer, $65 \%$ were satisfied that they may need less intensive chemotherapy based on their genomic test and often felt that the test had 'rescued' them from side effects. ${ }^{17,18}$ When the public were posed with a hypothetical situation of a life-threatening cancer diagnosis, $84 \%$ said they would not change their treatment approach if it was the only one available, even if PM indicated it was not going to work. ${ }^{19}$ Only $13 \%$ would accept that PM approach would predict treatment failure and so would not undertake the treatment. ${ }^{19}$

\section{Accuracy of tests}

Six papers cite the accuracy of PM testing as a benefit for patients. ${ }^{9,13,15,16,18,22}$ GEP tests are perceived to be accurate and trustworthy to inform chemotherapy decisions by $76 \%$ of patients. ${ }^{16}$ Qualitative research found that patients felt a benefit from using a 'new' and 'innovative' test, often without discussing test limitations with their doctor. ${ }^{13,18}$ Only $43 \%$ of patients knew that the test is not always correct. ${ }^{15}$ In clinical trials, $76 \%$ of patients had higher expectations of therapy as a result of testing, compared with the expected benefits described to them by their doctor. ${ }^{9,22}$

\section{Altruism}

Five papers found that patients feel positive about being involved in PM testing research in order to benefit others. ${ }^{9,12,18,20,22}$ All patients in a clinical trial interviewed reported wanting their experience to benefit others and $76 \%$ would be willing to undergo a new biopsy to assess a marker of interest. ${ }^{9,22}$ Of those undergoing somatic testing, $91 \%$ of patients would share their genomic cancer tests results for research purposes. ${ }^{12}$ Patients undergoing GEP testing were also positive that they were contributing to medical research. ${ }^{18,20}$

\section{Psychological impact of test results}

Five papers reported that the psychological impact of PM test results were a concern for patients. ${ }^{8,12,13,17,18}$ Of patients undergoing genomic sequencing, $32 \%$ felt a psychological burden while awaiting test results. ${ }^{8}$ Patients were most concerned that tests are not always part of routine investigations, which potentially delays results and the start of chemotherapy. ${ }^{12,13,17}$ Patients have also expressed anxiety related to the potential of a 'not interpretable' results, where an actionable mutation has not been found. $^{18}$

\section{Increased health insurance cost}

The potential for PM to increase health insurance costs was reported as a concern for both patients and the public in four papers. $^{12,17,19,22}$ Of members of the public in the USA, $40 \%$ believed that PM will significantly increase the cost of healthcare over the next 5 years. ${ }^{19}$ This is a concern for patients in the USA and Canada, but to a much lesser degree, reported by only $9 \%$ of those included. ${ }^{12,17,22}$ Fifteen per cent of patients found insurance discrimination as the top concern. ${ }^{12}$ Greater individual variability in concerns regarding insurance costs were found in other studies, with $27 \%$ of patients agreeing that it was a concern, $46 \%$ disagreeing and $27 \%$ were neutral. ${ }^{22}$

\section{Number of biopsies}

Four papers assessed the impact of biopsies on patients' opinion of PM. ${ }^{9,12,14,22}$ The greatest concern for those taking part in a PM clinical trial was the number of biopsies needed. ${ }^{22}$ Ninety-three per cent of patients would rather take part in a trial that required no new biopsies, if absolutely necessary, $39 \%$ would consent to one biopsy and $30 \%$ would consent to more than two biopsies. ${ }^{9,12}$ In China, $76 \%$ of patients would refuse any number of additional biopsies. ${ }^{12}$ Complications as a result of a biopsy were cited as the greatest factor for not pursuing testing. ${ }^{12}$ 


\section{Discussion}

We have demonstrated that both patients and the public are enthusiastic to engage in conversations regarding PM in cancer treatment. Patients have a greater understanding of PM terminology compared with members of the public. The extent of patient understanding, however, depends on the context in which they have been exposed to PM. Patients were often found to be unsure of how PM tests work and many experienced a psychological burden while awaiting and interpreting test results. Despite concerns, patients are more willing than the public to use genomic tests to inform cancer treatment. The public cite concerns of increasing healthcare costs as the main drawback of PM. This may be because if PM tests are not covered by the NHS or health insurance, they could be unaffordable if one was required to pay for the test privately. These findings also illustrate the impact of illness on psychology, with patients less concerned about cost, instead hopeful that the tests will improve outcomes despite many reporting a lack of understanding.

\section{Understanding and concerns of PM in patients}

The emergence of PM has led to an increasing number of clinical trials for genetic and genomic tests and treatments, particularly in cancer. ${ }^{23}$ Understanding of PM terminology was greater in patients enrolled in clinical trials, compared with those undergoing testing purely to inform their treatment. A recent study found that if members of the public are given an educational intervention prior to enrolment in a hypothetical cancer clinical trial, they express greater interest and increased likelihood of enrolling. ${ }^{24}$ Importantly, the education intervention increased participation of members of the public with lower levels of education the most. Two of the clinical trials in this review had a disproportionate number of patients with degree level of education or higher $(66 \%$ and $69.5 \%$ ). ${ }^{9,22}$ This may be because these patients are more likely to independently research trials beforehand and so are more likely to enrol.

This highlights the opportunity for education interventions in clinical trials to aid in patient understanding of PM. In return, more patients, particularly those who have lower level of education, may participate in such trials which will improve inclusivity and help further clinical research.

Such success with education in clinical trials could, therefore, be used as a template to aid in routinely educating patients undergoing PM tests, such as GEP and somatic testing. Patients have voiced concerns that doctors do not always explain PM terminology in a patient-friendly manner: 'Because I can't make sense of some of the terms, other people have to explain them to me. When the doctor explains it to me, he explains it so quickly that I immediately forget it again. ${ }^{21}$ A lack of health literacy not only hinders patients' ability to give informed consent, but also leads to poor adherence to treatment and worse health outcomes. ${ }^{25}$ This is important to address as it will undermine patients' and healthcare professional's ability to undertake shared decision making, one of the main aims that the NHS has set out regarding $\mathrm{PM}^{1}{ }^{1}$

Simple education interventions (such as a picture book or video) to explain PM terminology, facilitated by a genetic counsellor, increases patient knowledge significantly. ${ }^{10}$ Genetic counsellors typically help patients understand and assess risk of inheriting or passing on a medical condition. ${ }^{26}$ The vast majority of cancer is not inherited and many of these patients undergo genomic testing of their cancer to inform treatment and prognosis. Patients and the public struggle to understand the difference between inherited genetic risk testing and genomic testing to confer prognosis or guide treatment. These are complex scientific concepts, with distinctions between them that even trained healthcare professionals often find difficult to understand.

The role of the genetic counsellor, therefore, needs to evolve to incorporate non-genetic specialisms, help to educate patients with non-inherited cancers and train colleagues in the multidisciplinary team on how to do so. This process has already begun, with the NHS in the UK proposing a change in title from 'genetic counsellor' to 'genomic counsellor' to reflect the emphasis on testing noninherited conditions. ${ }^{27}$ While this may have little impact when understanding of genomics is low, the work of genomic counsellors to help educate patients has the potential to improve awareness and understanding of the concept.

Patients undergoing PM testing for cancer also experience considerable psychological concerns while awaiting and interpreting test results. It could be argued that a lack of understanding of how genomic tests are used to inform treatment contributes to the anxiety some patients experience. Genomic counsellors may help to address concerns by educating patients on the implication of potential PM test results. Alongside the development of infrastructure for diagnosing cancer faster and more effectively via rapid diagnostic centres, this education and more efficient diagnostic pathway will help to ease the integration of PM into standard clinical practice. ${ }^{28}$

\section{Understanding and the concerns of PM of the public}

Members of the public were found to be largely unfamiliar with the term PM in cancer, and this lack of awareness is a major barrier to the integrating PM within healthcare systems. ${ }^{2}$ The difficulties that the public have in understanding PM is not confined to cancer and has been found in other contexts; for example, a study of parents with a child undergoing PM testing reported that a proper explanation of genomic testing from healthcare professionals is the most important issue that needs to be addressed. ${ }^{29}$

Despite a lack of knowledge, the systematic review by Holden et al found that the public are enthusiastic about the idea of integrating PM into standard clinical practice. ${ }^{3}$ This is particularly true in studies that observed positive public opinion of PM use in conditions such as asthma and depression. ${ }^{30,31}$ In the context of informing cancer treatment, however, the public are less likely to use the results of PM testing to inform cancer treatment compared with patients if the test meant foregoing treatment that will not be effective. ${ }^{19}$ This hesitation shows a lack of trust in the evidence for genomic testing, which would limit the full potential of a PM approach to healthcare.

For the members of the public who have not personally experienced a cancer diagnosis, they may believe they would be more likely to choose high levels of chemotherapy without considering the side effects of these medications. The reluctance to incorporate PM into cancer treatment may, therefore, stem from a lack of exposure to the topic and subsequent lack of understanding of the potential benefits of PM. Greater education of, and exposure to, PM is needed for the public to be able to trust in genomic testing methods. Lower uptake of genomic tests will mean increased NHS spending on potentially less-effective treatments and delays in finding the right treatment for patients. 
Genome UK's 2020 initiative states: 'We will commit to communicating openly with patients and the public and make increasing understanding of genomics and health literacy centra to our communications and outreach approach over the next 10 years. ${ }^{1}$ Despite clear intentions from the UK government, there is little evidence of dialogue with the public on PM or provision of educational material. The NHS website's information for cancer does not mention the possibility of genomics to aid in cancer diagnosis or treatment. It could be argued that without the availability of patient-friendly educational material on PM in cancer, knowledge and understanding will be slow to change and concerns will not be addressed.

Education and support for patients is, however, available from charities such as Cancer Research UK, Macmillan Cancer Support and Breast Cancer Now, all of which provide education on PM and how this can impact someone's care on their websites. These are valuable resources that healthcare professionals can signpost to patients to improve understanding and offer local support to patients via groups affected by the same condition.

\section{Differences in understanding and concerns according to population}

The public from the USA were more concerned than patients that $\mathrm{PM}$ in cancer will cause an increase in health insurance costs. This has been found in numerous studies of public opinion of PM in other illnesses in the USA, Europe, Asia and Australia, regardless of the year of publication. ${ }^{3}$ This is a valid concern for patients and the public as high costs of PM research and clinical trials, and low volume of drug sales can drive up prices of cancer treatments with the knock-on effect to insurance costs in those countries. For this concern to be addressed, healthcare policy needs to provide equal and affordable access to new testing and treatments, although in countries such as the USA, healthcare costs are a politically divisive subject. The public can also be given reassurance that PM has the potential to lower healthcare costs by decreasing incidents of adverse drug reactions, providing early diagnosis of more effective treatment of disease.

There is also evidence of difference in concerns regarding PM testing between races. ${ }^{21}$ Holden et al identified that people from ethnic minority backgrounds are less willing to use PM in their care and have more concerns with its use, compared with non-minority populations. ${ }^{3}$ Such concerns regarding the use of PM in the care for people in ethnic minority groups may stem from discrimination to access to treatment. ${ }^{3}$ Ethnic minority populations are consistently underrepresented in PM research despite the majority $(57 \%-97 \%)$ being willing to participate in such research. ${ }^{32}$ Future studies must ensure minority groups are fairly represented in PM research, and dialogue between organisations (such as the NHS) and members of ethnic minority groups must happen to ensure that concerns are recognised and acted upon.

\section{Conclusion}

As the evidence base for using PM in clinical practice expands, the public and patients must be supported in making decisions regarding their current and future care. Results of this review specifically suggest a role for a healthcare professional in supporting patients undergoing PM tests and treatment, which could be undertaken by genomic counsellors. This has wider implications to the healthcare professional workforce as there needs to be education and upskilling regarding PM. Currently, this issue is being address by a PGx working party, with input from key specialties, including oncology. As only one paper has been published relating to public opinion of PM in oncology, further research is needed in this area, particularly outside of the USA. The public's concerns regarding PM can be addressed with adequate healthcare policy ensuring equal access to PM for all, without significantly increasing healthcare costs.

\section{Supplementary material}

Additional supplementary material may be found in the online version of this article at www.rcpjournals.org/fhj:

S1 - Full search strategy.

S2 - Study characteristics and quality.

\section{References}

1 Department of Health and Social Care, Department for Business, Energy \& Industrial Strategy, Office for Life Sciences, Lord Bethell of Romford. Genome UK: the future of healthcare. GOV.UK, 2020. [Accessed 29 October 2020]. www.gov.uk/government/publications/ genome-uk-the-future-of-healthcare

2 Smittenaar CR, Petersen KA, Stewart K, Moitt N. Cancer incidence and mortality projections in the UK until 2035. Br J Cancer 2016; 115:1147-55.

3 Holden C, Bignell L, Mukhopadhyay S, Jones C. The public perception of the facilitators and barriers to implementing personalized medicine: A systematic review. Per Med 2019;16:409-20.

4 Day S, Coombes RC, McGrath-Lone L, Schoenborn C, Ward H. Stratified, precision or personalised medicine? Cancer services in the 'real world' of a London hospital. Sociol Health IIIn 2017:39:143-58.

5 Lerman C. BRCA1 testing in families with hereditary breast-ovarian cancer. JAMA 1996;275:1885.

6 Hong QN, Fàbregues S, Bartlett G et al. The Mixed Methods Appraisal Tool (MMAT) version 2018 for information professionals and researchers. Education for Information 2018;34:285-91.

7 Giuse NB, Kusnoor SV, Koonce TY et al. Guiding oncology patients through the maze of precision medicine HHS public access. J Heal Commun 2016;21(Suppl):5-17.

8 Gray SW, Hicks-Courant K, Lathan CS et al. Attitudes of patients with cancer about personalized medicine and somatic genetic testing. J Oncol Pract 2012;8:329-35.

9 Lemech C, Dua D, Newmark J et al. Patients' perceptions of research biopsies in phase I oncology trials. Oncology 2015;88:95-102.

10 McDaniels BA, Hianik RS, Bellcross C et al. The impact of genetic counseling educational tools on patients' knowledge of molecular testing terminology. J Cancer Educ 2020;35:864-70.

11 Rogith D, Yusuf RA, Hovick SR et al. Patient knowledge and information-seeking about personalized cancer therapy. Int J Med Inform 2016;88:52-7.

12 Blanchette PS, Spreafico A, Miller FA et al. Genomic testing in cancer: Patient knowledge, attitudes, and expectations. Cancer 2014;120:3066-73.

13 Bombard Y, Rozmovits L, Trudeau M et al. Patients' perceptions of gene expression profiling in breast cancer treatment decisions. Curr Oncol 2014;21:e203-11.

14 Ciardiello F, Adams R, Tabernero ] et al. Awareness, understanding and adoption of precision medicine to deliver personalized treatment for patients with cancer: a multinational survey comparison of physicians and patients. Oncologist 2016;21:292-300.

15 Lipkus IM, Vadaparampil ST, Jacobsen PB, Miree CA. Knowledge about genomic recurrence risk testing among breast cancer survivors. J Cancer Educ 2011;26:664-9. 
16 Richman AR, Tzeng JP, Carey LA, Retèl VP, Brewer NT. Knowledge of genomic testing among early-stage breast cancer patients. Psychooncology 2011;20:28-35.

17 Bombard Y, Rozmovits L, Trudeau M et al. Access to personalized medicine: factors influencing the use and value of gene expression profiling in breast cancer treatment. Curr Oncol 2014;21:e426-33.

18 Pellegrini I, Rapti M, Extra J-M et al. Tailored chemotherapy based on tumour gene expression analysis: breast cancer patients' misinterpretations and positive attitudes. Eur J Cancer Care (Engl) 2012;21:242-50.

19 Garfield S, Douglas MP, Macdonald KV, Marshall DA, Phillips KA. Consumer familiarity, perspectives and expected value of personalized medicine with a focus on applications in oncology. Per Med 2015;12:13-22.

20 Halverson CME, Clift KE, McCormick JB. Was it worth it? Patients' perspectives on the perceived value of genomic-based individualized medicine. J Community Genet 2016;7:145-52.

21 Perry ], Wöhlke S, Heßling AC, Schicktanz S. Why take part in personalised cancer research? Patients' genetic misconception, genetic responsibility and incomprehension of stratification-An empirical-ethical examination. Eur J Cancer Care (Engl) 2017;26: $1-12$.

22 Kuderer NM, Burton KA, Blau S et al. Participant attitudes toward an intensive trial of multiple biopsies, multidimensional molecular analysis, and reporting of results in metastatic triple-negative breast cancer. JCO Precis Oncol 2017;1:PO.17.00076.

23 Garralda E, Dienstmann R, Piris-Giménez A et al. New clinical trial designs in the era of precision medicine. Mol Oncol 2019;13:549-57.

24 Mancini J, Briggs A, Elkin EB et al. The impact of patient education on consideration of enrollment in clinical trials. J Community Support Oncol 2018;16:e81-8.
25 Berkman ND, Sheridan SL, Donahue KE, Halpern DJ, Crotty K. Low health literacy and health outcomes: An updated systematic review. Ann Intern Med 2011;155:97-107.

26 Patch C, Middleton A. Genetic counselling in the era of genomic medicine. Br Med Bull 2018;126:27-36.

27 NHS. Genomic counselling. NHS, 2018. www.healthcareers.nhs. uk/explore-roles/healthcare-science/roles-healthcare-science/lifesciences/genomic-counselling [Accessed 21 March 2021].

28 NHS England, NHS Improvement. Rapid Diagnostic centres vision and 2019/20 implementation specification. NHS, 2019.

29 Zhang SC, Bruce C, Hayden M, Rieder MJ. Public perceptions of pharmacogenetics. Pediatrics 2014;133:e1258-67.

30 Rogausch A, Prause D, Schallengerb A, Brockmöller J, Himmel W. Patients' and physicians' perspectives on pharmacogenetic testing. Pharmacogenomics 2006;7:49-59.

31 Barr M, Rose D. The great ambivalence: Factors likely to affect service user and public acceptability of the pharmacogenomics of antidepressant medication. Sociol Heal IIIn 2008;30:944-58.

32 Fisher ER, Pratt R, Esch $R$ et al. The role of race and ethnicity in views toward and participation in genetic studies and precision medicine research in the United States: A systematic review of qualitative and quantitative studies. Mol Genet Genomic Med 2020;8:e1099.

Address for correspondence: Mr Jed Botham, Audrey Emerton Building, Eastern Road, Kemptown, Brighton BN2 OAE, UK.

Email: j.botham1@uni.bsms.ac.uk 